\title{
Mitotic and salivary gland chromosome analyses in the Musca domestica L. (house fly) (Diptera: Muscidae)
}

\author{
M. EL AGOZE,* F. LEMEUNIER, \& G. PERIQUET \\ Institut de Biocénotique Expérimentale des Agrosystèmes. Faculté des Sciences, Parc Grandmont, 37200 Tours, France
}

\begin{abstract}
The mitotic chromosomes in Musca domestica consist of five pairs of autosomes and an X, Y sex chromosome pair. They respond to C-banding with procentric bands on all autosomes and deep staining over most of the $\mathrm{X}$ and $\mathrm{Y}$ chromosomes. Polytene chromosomes were previously found in several larval and pupal tissue of Musca domestica. Polytene chromosome reference maps of the two sexual and the five autosomal chromosomes of Musca domestica from salivary gland cells are shown. Characteristic features of each chromosome are described identifying areas that are difficult to analyse.
\end{abstract}

Keywords: evolution, karyotype, Musca domestica, population.

\section{Introduction}

Musca domestica (house fly) has been known for several years to play an important role as a pest insect in several parts of the world. The increased development of strains resistant to insecticide treatments has led to a better knowledge of the genetics of the fly but its cytogenetics remain to be described. The genus Musca includes the domestica complex, along with several other species. This complex contains several intergrading forms which differ according to their biogeographical distribution (Milani, 1975). Within the domestica complex, the different forms have been described according to morphological, geographical, chromosomal data and to hybridization tests. Milani (1967) reported that several traits used for taxonomical purposes, e.g. front width and abdominal colour pattern, are modified both by developmental conditions and by their genetic background, and thus cannot be used as unconditional criteria for species identification.

Previous reports on the cytology of Musca domestica have described the basic mitotic karyotype (Ramade, 1961; Boyes, 1967; Milani, 1967) and have tried to map the polytene chromosomes both in the salivary gland of larvae (Sharma et al., 1979) and in the bristle-

Correspondence: F. Lemeunier. Laboratoire de Biologie et Génétique Evolutives, CNRS, 91198 GIF/Yvette Cedex, France.

*Present address: Department of Entomology, Faculty of Sciences Ain Shams University, Abassia, Cairo, Egypt. forming cells of the thoracic epidermis of the pupae (Kaur \& Kaur, 1982). None of the previous reports had indicated the full potential for cytogenetic analysis of these chromosomes. A complete description of the chromosomes is an essential aid to genetic studies and to understanding the organization of chromosomes in heterochromatin and euchromatin regions.

Aquaintance with mitotic chromosomes is especially interesting in describing sex chromosomes which usually have large amounts of heterochromatin (Bedo, 1980; Gatti \& Pimpinelli, 1983; Pimpinelli et al., 1976), while workable polytene chromosomes allow an accurate analysis of euchromatin regions.

The object of this paper is to present the main features of each mitotic and polytene chromosome found in a standard European house fly culture, Musca domestica L. (Sacca, 1967), which has the normal X and $Y$ sex-chromosomes and has no autosomal sexual determinants, in order to provide a useful step towards the understanding of the phylogenetic inter-relationship between the different forms of Musca domestica complex and to facilitate genetic studies of this species which is now among the genetically best known insects (Milani, 1975).

\section{Materials and methods}

Musca domestica used in this study were WHO standard reference strain (OMS Procida, Marseille) generously provided by Dr Bergé, INRA, Antibes, France. Adult flies were kept in $45 \times 45 \times 45 \mathrm{~cm}$ plastic 
cages and were fed on dry milk. After the fifth day of life until their death, they were provided daily with a fresh larval artificial medium on which they could lay their eggs. The larval medium, composed of 8 per cent dry milk, 8 per cent wheat bran, 3 per cent dry yeast and 8 per cent tissue paper in tap water, was placed in plastic boxes filled to a third of their height with about $1 \mathrm{~g}$ of medium per larva. After oviposition, the boxes were labelled and covered with heavy muslin. Cultures were maintained at a temperature of $28^{\circ}+2^{\circ} \mathrm{C}$ with 75 per cent relative humidity and a $16 \mathrm{~h}$ light $-8 \mathrm{~h}$ dark cycle.

\section{Mitotic chromosomes}

Mitotic chromosome preparations were made from brain ganglia of late third instar larvae. We used the airdrying technique, first described by Guest \& Hsu (1973) for D. melanogaster, then used with slight modifications by Bedo (1980) for Lucilia cuprina and by Zacharopoulou (1987) for Ceratitis capitata.

Chromosome slide preparation. Air-dried neuroblast preparations of late third instar larvae were routinely used. Larval brains were dissected in 1 per cent sodium citrate and treated in this hypotonic solution for 10-15 min before fixing in fresh ethanol-acetic acid mixture (3:1) for 2-3 min. The fixed tissue was then transferred to a drop of 60 per cent acetic acid on a clean slide and finely macerated forming a cell suspension. Finally the slide was placed on a warm hotplate (surface temperature $45-50^{\circ}$ ) and the drop of cell suspension was allowed to evaporate while intermittently moving it about the slide surface.

C-Banding. Slides were treated for $0.5 \mathrm{~h}$ at room temperature in $0.1 \mathrm{~N} \mathrm{HCl}$, then treated in saturated $\mathrm{Ba}(\mathrm{OH})_{2}$ at $50^{\circ} \mathrm{C}$ for $5 \mathrm{~min}$. This was followed by washing in running tap water, rinsing twice in distilled water, and air drying at room temperature in a slide rack. The slides were then incubated in $2 \times \mathrm{SSC}$ at $60^{\circ} \mathrm{C}$ for $1 \mathrm{~h}$, washed and stained in 3 per cent Giemsa in phosphate buffer ( $\mathrm{pH} 6.8$ ) for $1 \mathrm{~h}$, washed, and finally air dried.

Hoechst 33258 staining. Air-dried preparations were stained for $10 \mathrm{~min}$ in a solution of $2 \mu \mathrm{g} / \mathrm{ml}$ Hoechst in pH 7 Mcllvaine buffer. The slides were then washed and, for fluorescence observation, a coverslip was applied with distilled water.

\section{Salivary gland chromosomes}

Active third instar larvae (just before pupation) were dissected in 45 per cent acetic acid. The salivary glands were isolated and transferred into the well of a depres- sion slide containing $1 \mathrm{~N} \mathrm{HCI}$ for 1 min of hydrolysis. The glands were then transferred to a microscope slide and a drop of lacto acetic acid (1 part lactic acid, 2 parts 60 per cent acetic acid) was added for $5 \mathrm{~min}$ of fixation.

The nuclear membranes were torn with dissecting needles following fixation to allow the spread of the chromosomes, and a drop of 2 per cent lacto acetic orcein was added. After 15 min of staining at $20^{\circ} \mathrm{C}$, the sample was squashed in a drop of the same lacto acetic acid after applying a clean coverslip. Because the salivary gland chromosomes of the house fly tend to remain entangled in a mass, it was necessary to press firmly on the coverslip, in addition to the gentle tapping necessary for spreading, using several layers of blotting paper to absorb excess stain. Coverslips were sealed with nail polish. Slides thus prepared can be stored in a refrigerator for about 1 month. Observations and photographs were performed using a Zeiss $63 \times$ planapochromat phase-contrast objective. Prints showing the best morphology for each chromosome region were selected and used to construct composite maps of each chromosome.

\section{Results and discussion}

\section{Mitotic chromosomes}

As reported by Milani et al. (1967), the mitotic karyotype of Musca domestica has six pairs of chromosomes including an $\mathrm{XY} / \mathrm{XX}$ chromosome pair, which appears somewhat darker than the autosomes after conventional staining of metaphase figures by Giemsa or Orcein. According to previous studies and to the nomenclature proposed by Levan et al. (1964) the five autosomal pairs are meta- or submetacentric (Fig. 1).

The $\mathrm{X}$ and $\mathrm{Y}$ chromosomes are metacentric, with the $\mathrm{Y}$ having about one-third of the $\mathrm{X}$ length. The $\mathrm{Y}$ is thus the shortest member of the complement. Moreover, the $\mathrm{X}$ chromosome has the same length as the first autosomal metacentric pair. The longest autosomal pair often has a secondary constriction in the middle of one of its arms, which agrees with Boyes (1967).

All the autosomes show a similar C-banding pattern with thin, darkly stained bands on either side of the non-staining centromeric constriction, in prophase, and extended early metaphase chromosomes (Fig. 2). These procentric bands are very sensitive to variations in the banding procedure, often showing less clearly on some autosomes or staining only on one side of the centromere. This phenomenon has also been described by Bedo (1980) in Lucilia cuprina.

Contrary to the autosomes, the sex chromosomes are markedly stained. The X chromosome is dark, with 
Fig. 1 Mitotic chromosomes of $M$. domestica male stained with Giemsa (a) Male karyotype of M. domestica after staining with Hoechst (b).
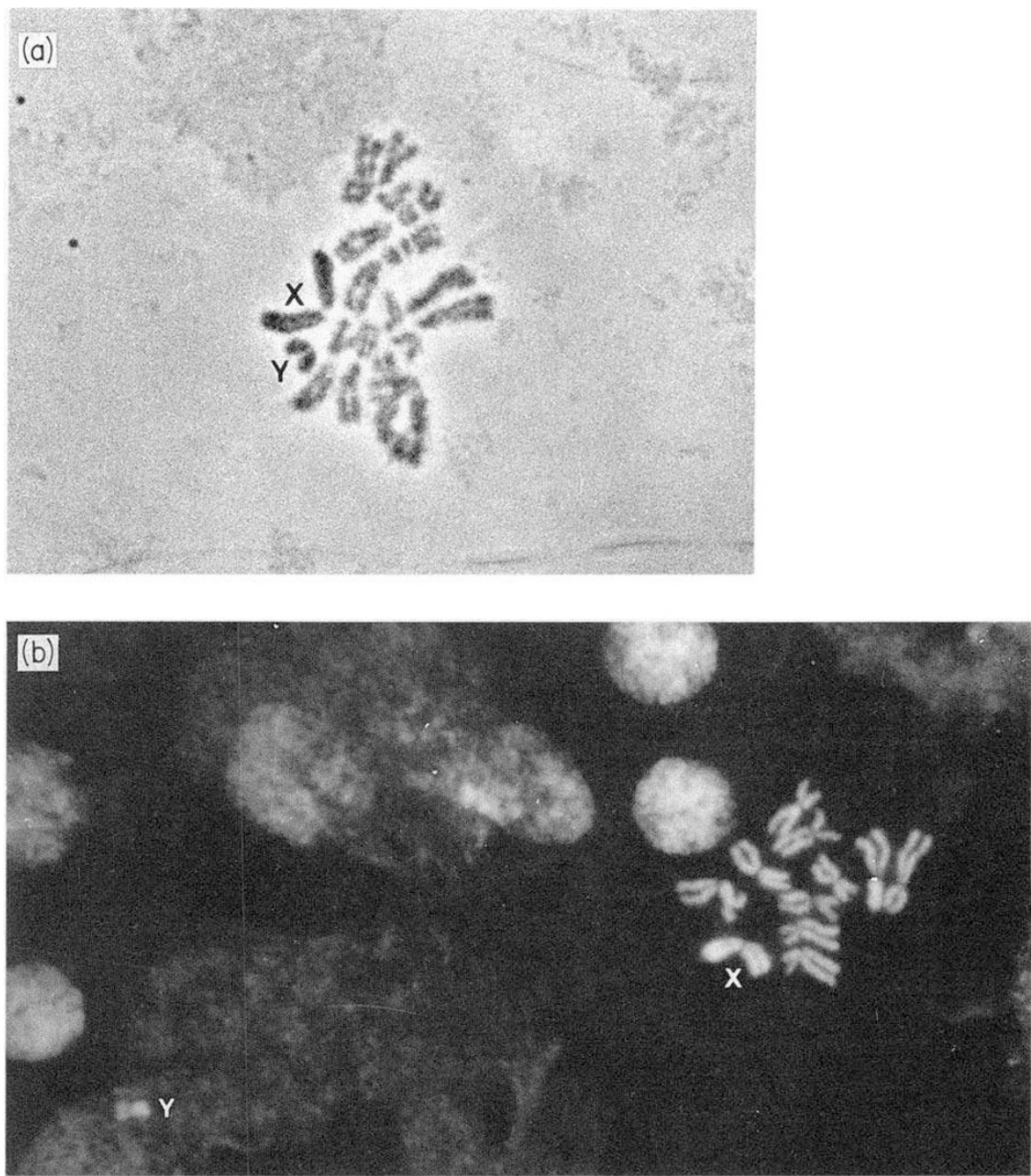

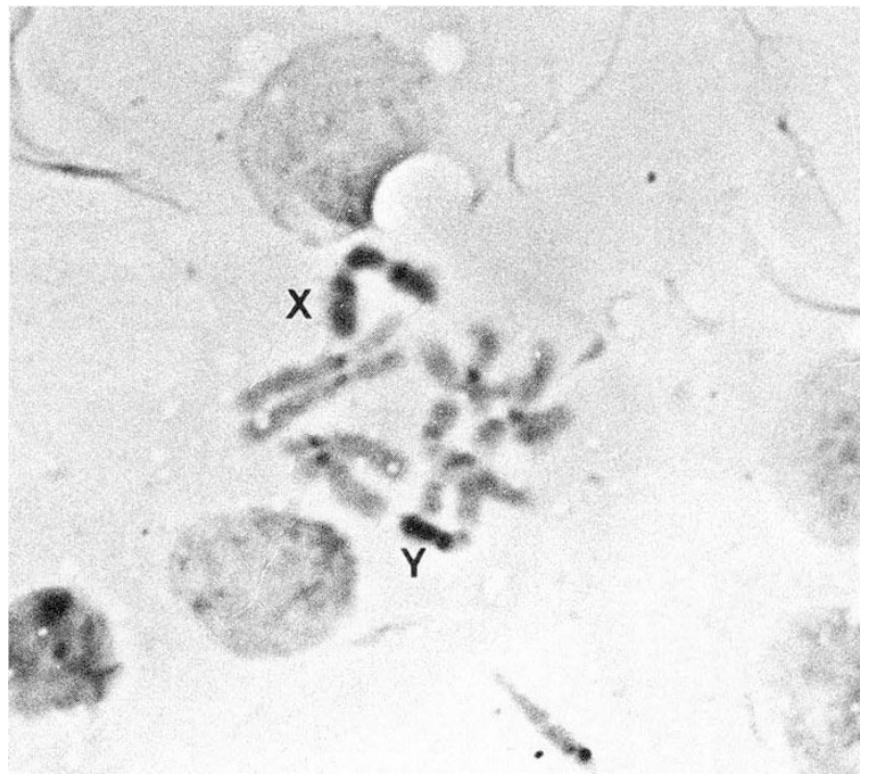

Fig. 2 Mitotic chromosomes of M. domestica showing C-banding. one dark region on each side of the non-staining centromere, followed by a short, light region and a distally darker region on each arm of this metacentric chromosome. The $\mathrm{Y}$ chromosome is usually uniformly dark after C-banding. Hoechst 33258 produces fluorescence of all autosomes without prominent bands, while the sex chromosomes fluoresce somewhat brighter than the autosomes. Ullerich (1976), in four Chrysomya species, and Bedo (1980), in Lucilia cuprina, found autosomal C-banding patterns similar to those reported here for Musca domestica. The differences observed here occur in sex chromosome C-banding.

\section{Polytene chromosomes}

The polytene chromosomes of $M$. domestica are generally characterized by a clear banding pattern suitable for identification. Nevertheless, high levels of ectopic pairing, break points and fragmentation occur very often (Vecchi \& Rubini, 1973; Sharma et al., 1979). 
However, using our preparation techniques, similar to those used by Zacharopoulou (1987) (with slight modifications) for the preparation of Ceratitis capita salivary gland chromosomes, we obtained results that were sufficient for analysis and photographs, and that offered reasonable assurance as to the suitable quality of house fly salivary gland chromosomes for use as good cytological material.

In $M$. domestica salivary gland, and unlike Drosophila melanogaster (Lefevre, 1976) and some species of Chironomus (Sorsa, 1988), there is no evidence of the presence of a chromocentre binding together nonhomologous chromosomes at their centric heterochromatin; however, some ectopic pairing may exist. The presence of several break points and constrictions make it difficult, for the moment, to determine the exact position of centromeres. However, in this paper, subject to further studies for confirmation, we determined the probable positions of centromeres according to the characters used by Foster et al. (1980) and Bedo (1987) for determining the same structure in Lucilia cuprina and Ceratitis capitata, respectively.

The polytene complement consists of 10 autosomal arms and two heterosomes ( $\mathrm{X}$ and $\mathrm{Y}$ ). This study does not show the correlation between the polytene and mitotic chromosomes. Differences between preparations from male and female cells indicated the shape of the $\mathrm{Y}$ chromosome, as in the culture used here. The $\mathrm{Y}$ chromosome is sex determining whereas intense heterochromatin expression characterizes the $\mathrm{X}$ chromosome. In the house fly, as in other members of the order Diptera, the two heterosomes are usually found near each other. We have adopted a nomenclature system in which the heterosomes are considered as the first pair while the autosomes are arranged in ascending order of length. The charac- teristic ends of the chromosomes aid in their identification, and, to prevent future confusion, the entire complement is referenced by map division numbers. It is divided into 36 sections, each section subdivided into three or four subsections (named $a$ to $d$ ). Each subsection starts by a characteristic band. The chromosomes are referenced as follows: $\mathrm{Y}(1-4), \mathrm{X}(5-10)$, II (11-14), III (15-19), IV (20-24), V (25-30), VI $(31-36)$.

We have not studied the evolution of the puffing pattern according to the different larval stages, but have described that of the larva just before pupation. The disturbance in pairing of homologous chromosomes was also noted.

Chromosome $Y$ (Fig. 3). This chromosome can be easily recognized as it is the smallest of the complement, usually found unbroken and marked by its general heterochromatic aspect with few clear bands. The free end of this chromosome in $1 \mathrm{~A}$ is almost round and is flanked at its distal margin (1a) by a dark band. The dark band at the other margin (1b), preceded by a dotted one, characterizes this end. Sections 1 and 2, which are separated by a constriction (2a), are distinguished by a bundle of diffused bands; the heavy dark band in $2 \mathrm{~b}$ followed by a probable centromere in $2 \mathrm{c}$ is typical. Section 3 , characterized by its flattened portion, is flanked by a dark band in 3a. The doublet of dark bands in $3 \mathrm{c}$ forms an excellent landmark for the identification of this chromosome. The semicircular end in $4 c$ is recognizable by the bundle of dark bands at its tip.

Chromosome $X$ (Fig. 3). This chromosome is usually found apart, probably because of slight ectopic pairing with the rest of the complement. It is distinctive within
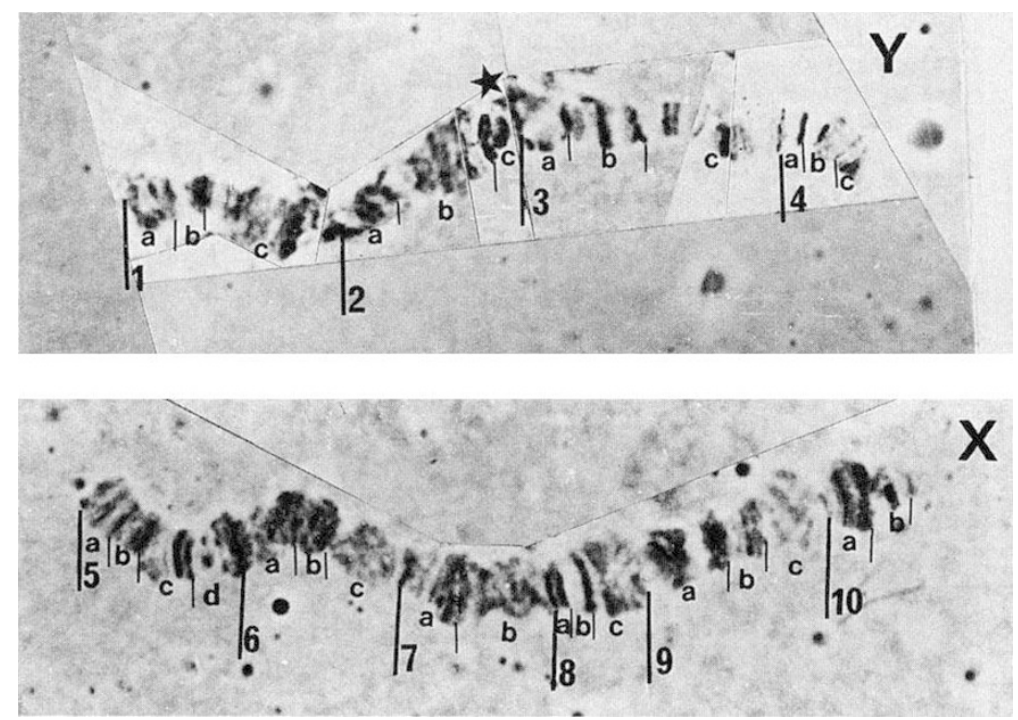

Fig. 3 Polytene chromosome $\mathrm{Y}$ and $\mathrm{X}$. 
the complement as it is found unbroken, with its characteristic tip. The $5 \mathrm{a}$ end is semi-flared with three doublets of moderate and dark bands. The distal band of the first doublet is dotted and dark and may change in shape (see Fig. 6). The doublet of heavy dark bands in $5 c$ is easily recognizable. Sections 6-7 are characterized by their heterochromatic aspects with diffuse light and dark bands.

Section 8 is the most distinctive landmark for the identification of chromosome $\mathrm{X}$. Each subsection is clearly identifiable: $8 \mathrm{a}$ with its doublet of heavy dark bands; $8 \mathrm{~b}$ with its flattened portion flanked by a heavy dark band; and the puff in $8 \mathrm{c}$ with its diffuse light and moderate bands. Section 9 is divided into three subsections: 9a with two bundles of diffused dark bands; $9 \mathrm{~b}$ has three dark bands; and $9 \mathrm{c}$ is recognizable by its diffuse moderate bands.

Section 10:10a is another excellent landmark for this chromosome; it is separated by a clear constriction from $10 \mathrm{~b}$. The distal end in $10 \mathrm{~b}$ is characterized by a heavy dark band at its base and a faint stained triangle at its tip.

Chromosome II (Fig. 4). The semicircular end of this chromosome, in $11 \mathrm{a}$, is flanked by a dotted dark band. A doublet of heavy bands in $11 \mathrm{~b}$ is characteristic and, with the flattened area followed by the dotted heavy band in $11 \mathrm{c}$, provides a good landmark. $11 \mathrm{~d}$ is separated from $11 \mathrm{c}$ by a constriction and from 12 by a probable centromere. $11 \mathrm{~d}$ is comprised of a series of light, moderate and dark bands which are sometimes associated ectopically. Section 12 is peculiar, with several characteristic puffs $(12 \mathrm{a}, 12 \mathrm{~b}, 12 \mathrm{c}, 12 \mathrm{~d})$ separated from each other by slight constrictions. Section 13 is an excellent landmark for chromosomes II. It starts by a constriction (13a) and ends by a prominent puff in 13c, flanked by a heavy dark band, typical for its recognition. Subsection $14 \mathrm{a}$ is also a good site for easy identification with a doublet of dark bands followed by a characteristic heavy one. $14 \mathrm{~b}$ may be identified by its
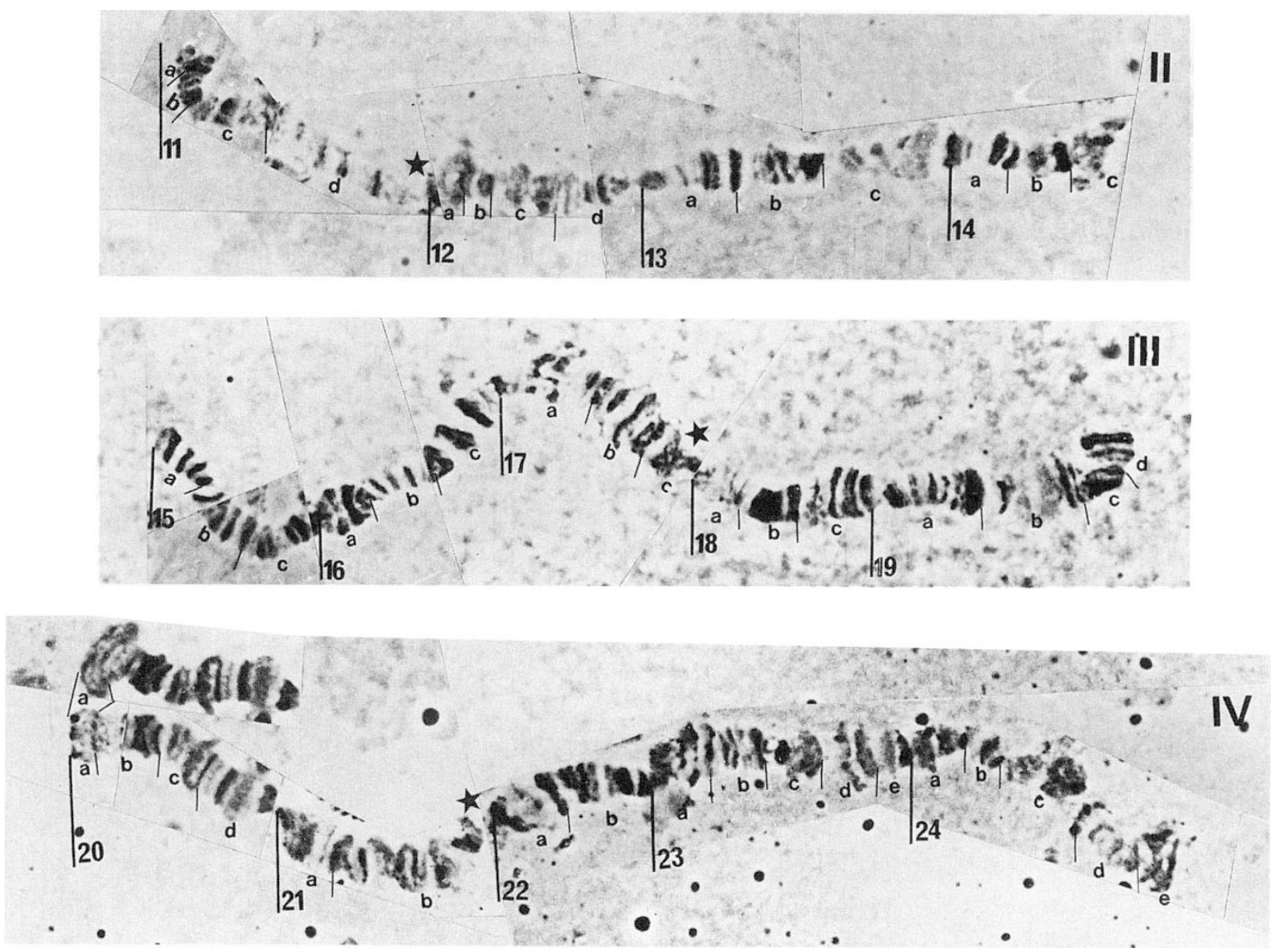

Fig. 4 Polytene chromosomes II, III and IV. 
bundle of dark bands. The semi-flared tip in 14c sometimes shows ectopic pairing, probably because of its heterochromatic composition.

Chromosome III (Fig. 4). This chromosome is generally identified by its darkly stained bands all throughout its length. In subsection 15a, the bands are arranged gradually according to size in a semi-flared end; the largest situated at the far portion. The puff in 16a is a good landmark, while subsection $16 \mathrm{~b}$ is flanked on each side by a constriction. $16 \mathrm{c}$ is characterized by three thick, dark bands separated by two lightly stained interband areas. The puff in $17 \mathrm{~b}$ is typical, with its characteristic dark band in the middle. A probable centromere occurs between sections 17 and 18 . Several doublets of dark bands are typical of $18 \mathrm{~b}$. Subsection $18 \mathrm{c}$ is separated from 19 a by a constriction. A large puff in $19 \mathrm{~b}$ is also an excellent landmark. The tip (19d) is characterized by its faint band at the distal margin, preceded by a prominent doublet of dark bands.

Chromosome IV (Fig. 4). The tip, 20a, is semicircular, flanked at both sides by dotted black bands. The distal band is more prominent and may show asynapsis or polymorphism as shown in Fig. 4. The puff in $20 \mathrm{~b}$ is situated between a dark band on one side and a characteristic bundle of dark bands on the other. This puff provides an excellent landmark for the recognition of this chromosome. In addition, $20 \mathrm{c}$ is also peculiar because it begins with a clear area followed by a doublet of moderate bands and ends with a bow shaped puff. Subsection $20 \mathrm{~d}$ is separated from section 21 by a constriction, $21 \mathrm{a}$ is characterized by its typical large puff while $21 \mathrm{~b}$ appears as a succession of several small puffs. The constriction between $21 \mathrm{~b}$ and $22 \mathrm{a}$ is the most likely location of the centromere. The prominent puff in $22 \mathrm{a}$, with its dotted, dark band in the middle, is typical. $22 \mathrm{~b}$ is characterized by a series of dark bands and separated from 23a by a constriction. Subsection $23 \mathrm{~b}$ is an excellent landmark with its puff, while the puff in $23 \mathrm{~d}$ is also very peculiar. The small puffs in $24 \mathrm{a}$, $24 \mathrm{~b}$ and $24 \mathrm{c}$ are typical. The tip, at $24 \mathrm{e}$, is characterized by its two thick dark, bent bands.

Chromosome V (Fig. 5). The two dotted dark bands of the distal margin from subsection 25a are typical of this chromosome's telomeric end which is variable in expression (Fig. 6). Subsection 25 b has a characteristic puff with a heavy band immediately distal.

$25 \mathrm{c}$ provides an excellent landmark with its two dark outer bands enclosing a very clear area in the middle of the subsection. In addition, the two large puffs in $25 \mathrm{~d}$ and $26 \mathrm{a}$, flanked by constrictions, are very peculiar. The series of dark bands in $26 \mathrm{~d}$ is characteristic of section 26.

In subsections $27 \mathrm{~b}$ and $27 \mathrm{c}$, new series of dark bands provide good landmarks near the presumed centromere site at the 27-28 boundary. The large puff in $28 \mathrm{~b}$, with its doublet of characteristic diffused moderate and dark bands in its middle, is an excellent recognition site. A weak point separates section 28 from section 29 . Subsection 29 d is typical with its doublet of diffuse moderate bands followed by three or four
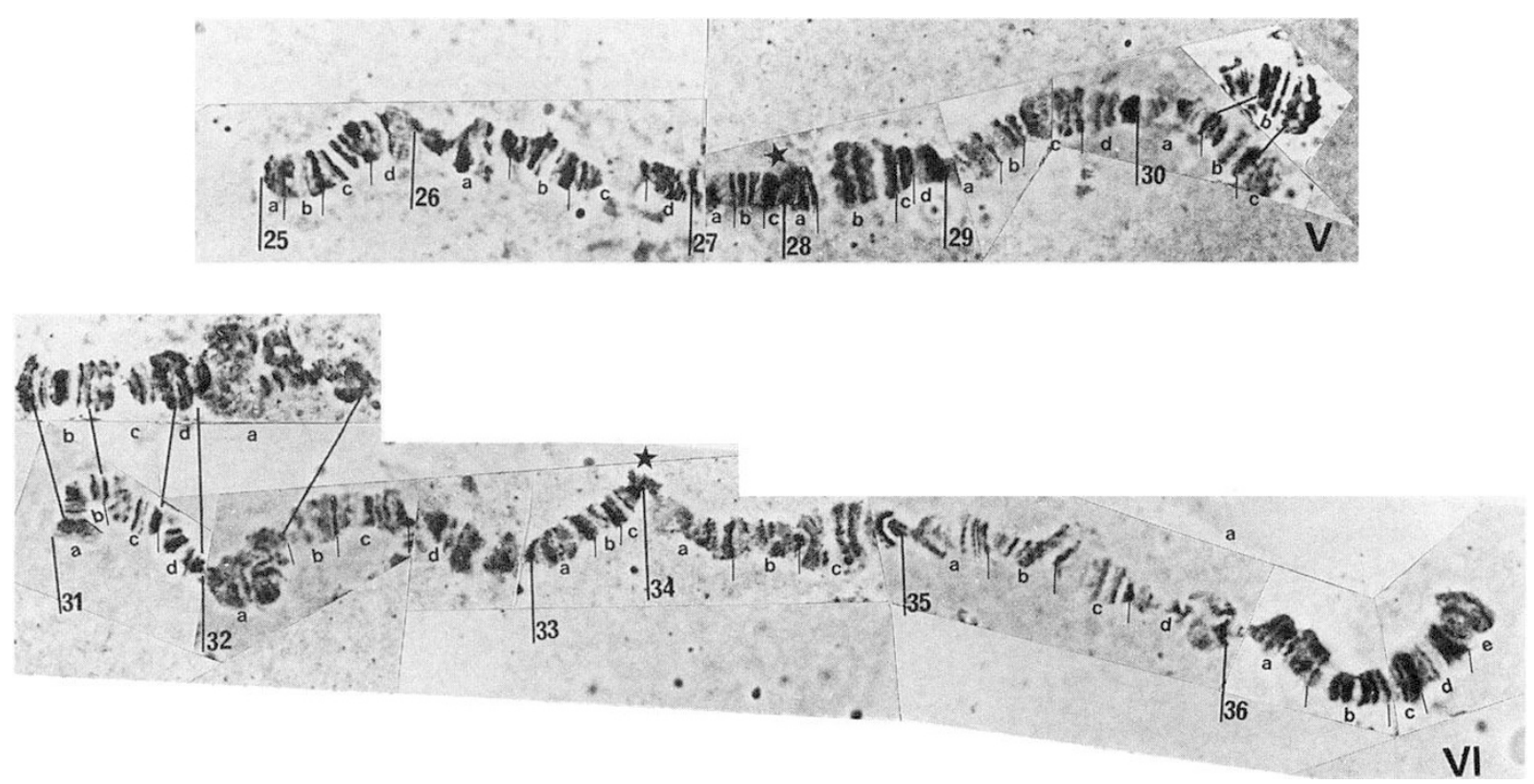

Fig. 5 Polytene chromosomes V and VI. 

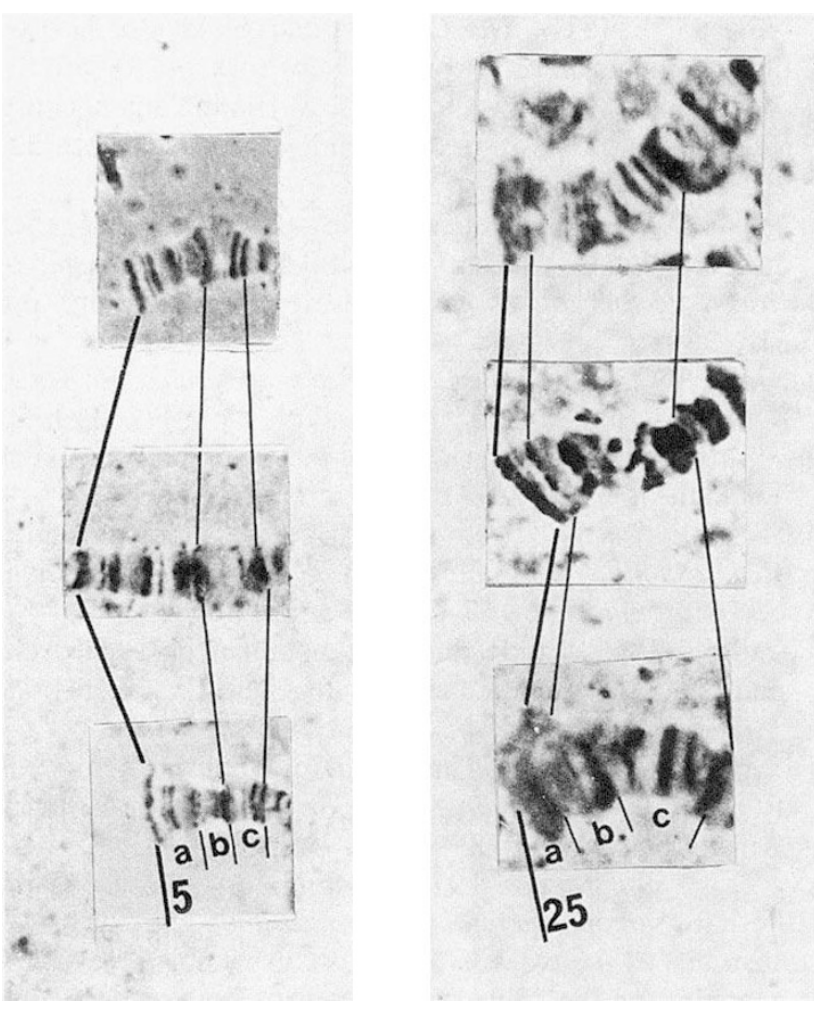

Fig. 6 Polymorphisms found at the tips of chromosomes $\mathrm{X}$ and $\mathrm{V}$.

smaller dark, thick bands. Subsection $30 \mathrm{c}$ is characterized by its semi-circular end.

Chromosome VI (Fig. 5). This chromosome is the longest of the complement; its semi-flared end in 31a is easily recognized by its thick terminal band preceded by a light band. The doublet of dark bands in $31 \mathrm{~b}$ is typical, sometimes with asynapsis as shown in Fig. 5. $31 \mathrm{c}$, with its small round puff with a dotted line is also peculiar. Subsection $31 \mathrm{~d}$ is separated from section 32 by a constriction.

Region $32 \mathrm{a}$ is frequently involved in ectoping pairing, producing a characteristic loop. Puffing is constantly expressed in subsection $32 \mathrm{~b}, 32 \mathrm{c}$ and $32 \mathrm{~d}$. Several characteristic small puffs are interspersed along section 33 , which is separated from section 32 by a constriction, the site of what is probably a centromere. Subsection $34 \mathrm{~b}$ usually shows asynapsis. The large puff in $34 \mathrm{c}$ is an excellent landmark. Section 35 is usually stretched with its puff $35 \mathrm{~d}$, difficult to describe but easily recognizable. Section 36 is also characteristic, with its numerous thick, dark bands. The telomeric end, $36 \mathrm{c}$, is fan shaped, flanked by a doublet of dotted dark bands.

\section{Conclusion}

The polytene chromosomes of Musca domestica described here provide good material for cytogenetic analysis and are of immediate practical use with workable photographic maps. Of course, the levels of resolution are not yet comparable to those achieved in Diptera, such as D. melanogaster, L. cuprina and $C$. capitata.

Because of the unknown homologies of most of the polytene chromosomes with the mitotic complement and the lack of certainty concerning centromere locations, some uncertainty remains in the nomenclature of both polytene and mitotic chromosomes. These results must be completed in order to obtain a more detailed polytene map and to provide a correlation between the genetic linkage group (known for some members of mitotic karyotype) (Wagoner, 1967) and the polytene chromosomes.

As in several members of the Diptera order, e.g. Sciara coprophila (Gabrusewycz-Garcia, 1964). Chironomus melanotus (Steinemann, 1978), Sarcophaga bullata (Samols \& Swift, 1979) and Lucilia cuprina (Bedo, 1982a), M. domestica heterosomes (X and Y) have polytene counterparts. In our studies, the size of the $\mathrm{X}$ chromosome in the diploid tissues was similar to that of the other autosomes, while it was much smaller in the salivary gland cells. These results suggest that, like Simulium ornatipes (Bedo, 1982b) and Chironomus melanotus (Steinemann, 1978), X heterochromatin in the Musca domestica is underreplicated in a pronounced pattern. Similar results were obtained in Drosophila (Heitz, 1934; Gall, 1973), Sarcophaga bullata (Samols \& Swift, 1979) and Lucilia cuprina (Bedo, 1982a). Thus it would now be interesting to undertake comparative studies to find the correlation that may exist between the sequence arrangement pattern of DNA organization and the under-replication of $\mathrm{X}$ heterochromatin in order to understand the evolution within the Diptera order.

\section{Acknowledgements}

The authors would like to thank Dr J. B. Bergé for supplying the Musca stock, Mrs C. Pouget and Mrs I. Gaspard for their help with the photography. We would also like to thank Dr S. Aulard for valuable discussions throughout the work. This work was supported by grants from the CNRS (URA 1298, UPA2411), the Ministère de l'Education Nationale (DRED - Evolution) and the programme Biotechno Centre.

\section{References}

BEDO, D. G. 1980. C, Q and H-banding in the analysis of Y chromosome rearrangement in Lucilia cuprina 
(Wiedemann) (Diptera: Calliphoridae). Chromosoma (Berl.), 77, 299-308.

BEDO, D. G. 1982a. Differential sex chromosome replication and dosage compensation in polytene trichogen cells of Lucilia cuprina (Diptera: Calliphoridae). Chromosoma, 87, 21-32.

BEDO, D. G. 1982 b. Pattern of polytene chromosome replication in Simulium ornatipes (Diptera: Simuliidae). Genetica (Den Haag), 59, 9-21.

BEDO, D. G. 1987. Polytene chromosome mapping in Ceratitis capitata (Diptera: Tephritidae). Genome, 29, 598-611.

BOYES, J. w. 1967. The cytology of Muscoid flies In: Wright, J. W. and Pal, R. (eds), Genetics of Insect Vectors of Disease. Elsevier, Amsterdam, 371-384.

BRIDGE 1935. Salivary chromosomal maps with a key to the banding of the chromosomes of Drosophila melanogaster, J. Hered. 29, 11-13.

FOSTER, G. G., WHITTEN, M. J., KONOVALOV, C., BEDO, D. G., MADDERN, R. H. AND BOON, D. J. 1980. Cytogenetic studies of Lucilia cuprina dorsalis R. D. (Diptera: Calliphoridae). Polytene chromosome maps of the autosomes and cytogenetic localisation of visible genetic markers. Chromosoma, $\mathbf{8 1}$, 151-168.

GABRUSEWYCZ-GARCIA, N. 1964. Cytological and autoradiographic studies in Sciara coprophila salivary gland chromosomes, Chromosoma, 15, 312-344.

GALL, J. G. 1973. Repetitive DNA. In: Drosophila in Molecular Cytogenetics. Plenum Press, New York.

GATTI, M. AND PIMPINELli, s. 1983. Cytological and genetic analysis of the $\mathrm{Y}$ chromosome of Drosophila melanogaster. I. Organisation of the fertility factors, Chromosoma, 88, 349-373.

GUEST, w. C. AND HSU, T. C. 1973. A new technique for preparing Drosophila neuroblast chromosomes. DIS, 50, 193-194.

HEITZ, E. 1934. Uber $\alpha$ - and $\beta$-Heterochromatin sowie Konstanz und Bau der chromomeren bei Drosophila, Biol. Zbl. 54, 588-609.

HSU, T. C. 1971. Heterochromatin pattern in metaphase chromosomes of Drosophila melanogaster, J. Hered., 62, 285-287.

KAUR, P. AND KAUR, G. 1982. Polytene chromosomes of $M u s c a$ domestica L. (Muscidae: Diptera). The Nucleus, 25, 123-129.

LEFEVRE, G. 1976. A photographic representation and interpretation of the polytene chromosomes of Drosophila melanogaster salivary glands. In: Ashburner $M$. and
Novitski, E. (eds), The Genetics and Biology of Drosophila, Academic Press, London, New York, pp. 31-66.

LEVAN, A., FREDGA, K. AND SANGBERG, A. A. 1964. Nomenclature for centromeric position on chromosomes. Hereditas, $\mathbf{5 2}$, 201-220.

MILANI, R. 1967. The genetics of Musca domestica and other muscoid flies. In: Wright, J. W. and Pal, R. (eds), Genetics of Insect Vectors of Diseases, Elsevier, Amsterdam, pp. 315-389.

MILANI, R. 1975. The house fly, Musca domestica: In: King, R. C. (ed.), Handbook of Genetics, Vol. 3, pp. 377-399.

MILANI, R., RUBINI, P. G. AND FRANCO, M. G. 1967. Sex determination in the house fly, Genetica Agraria, 21, 385-411.

PIMPINELLI, S., SANTIN1, G. AND GATTI, M. 1976. Characterisation of Drosophila heterochromatin. II. C and N-banding, Chromosoma (Berl.), 57, 377-386.

RAMADE, F. 1961. Etude du développement post-embryonnaire du testicule et de la spermatogènese chez l'asticot de Musca domestica L. Ann. Ins. Nat. Agron., 47, 1-63.

SACCA, G. 1967. Speciation in Musca. In: Wright, J. W. and Pal, R. (eds), Genetics of Insect Vectors of Diseases. Elsevier, Amsterdam. pp. 389-399.

SAMOLS, D. AND SwIFT, H. 1979. Genomic organisation in the flesh fly Sarcophaga bullata, Chromosoma, 75, 129-143.

SHARMA, G. P., HANDA, S. M., KAUR, P. AND CHOPRA, K. 1979. Mapping of the salivary gland chromosomes of Musca domestica - (Muscidae: Diptera), Cytologia, 44, 541-547.

SORSA, v. 1988. Polytene chromosomes in Genetic Research, Ellis Horwood Ltd, Chichester.

STEINEMANN, M. 1978. Co-replication of satellite DNA of Chironomus melanotus with main band DNA during polytenization, Chromosoma (Berl.), 66, 127-139.

ULlERICH, F. H. 1976. Chromosomenverhältnisse, konstitutives Heterochromatin und Geschlecht bestimmung, bei einigen Arten der Gattung Chrysomya (Calliphoridae: Diptera), Chromosoma (Berl.), 58, 113-136.

VECCHI, M. L. AND RUBINI, P. G. 1973. Polytene chromosomes of trichogen cells in Musca domestica L., Genet. Agraria. 27, 456-463.

WAGONER, D. E. 1967. Linkage group karyotype correlation in the house fly determined by cytological analysis of X-ray induced translocation, Genetics, 57, 729-739.

Zacharapoulou, A. 1987. Cytogenetic analysis of mitotic and salivary gland chromosomes in the Medfly Ceratitis capitata. Genome, 29, 67-71. 